\title{
Um ensaio teórico sobre a influência da Teoria da Troca Social e da Co-criação de Valor no Crowdfunding
}

A theoretical essay on the influence of Social Exchange Theory and Value Co-creation in Crowdfunding

Un ensayo teórico sobre la influencia de la Teoría del Intercambio Social y de la Co-creación de Valor en el Crowdfunding

DOI: https://doi.org/10.1590/1809-58442021112

\section{Camila da Silva Schmitt ${ }^{1}$}

https://orcid.org/0000-0002-8844-0136

\section{Martin de La Martiniere Petroll ${ }^{1}$}

https://orcid.org/0000-0001-7280-9202

${ }_{1}^{1}$ (Universidade Federal de Santa Catarina, Departamento de Administração, Programa de Pós-Graduação em Administração. Florianópolis - SC, Brasil).

\section{Resumo}

O presente ensaio teórico investigou o fenômeno do crowdfunding a partir da Teoria da Troca Social e da Co-criação de Valor no intuito de estabelecer proposições de pesquisas futuras. Percebeu-se que crowdfunding apresenta dinâmicas de co-criação em seus processos, onde os potenciais apoiadores - crowdfunders - envolvidos trabalham em estreita colaboração com o empresário de forma cooperativa, participando ativamente do desenvolvimento do projeto instaurado com feedbacks. Notou-se a plataforma crowdfunding como intermediário que facilita a interação repetida entre o proponente e apoiadores, criando valor não só financeiro. Mostrou que a criação e troca de novos conhecimentos na fase de inovação gera benefícios não financeiros ao projeto, uma vez que ganha a possibilidade de adaptar a proposta de valor ao mercado sem a necessidade de dispendiosas pesquisas. Conclui-se apresentando proposições de futuras pesquisas, como: concentrar esforços para analisar o conteúdo específico trabalhado em projetos bem-sucedidos de crowdfunding e compreender como pode auxiliar em estratégias de reciprocidade no contexto de troca social e co-criação de valor.

Palavras-chave: Teoria da Troca Social. Co-criação de Valor. Crowdfunding. Financiamento Colaborativo. Crowdfunders.

\section{Abstract}

The present theoretical essay investigated the phenomenon of crowdfunding from the Social Exchange Theory and Value Co-creation in order to establish future research proposals. It was 
noticed that crowdfunding presents co-creation dynamics in its processes, where the potential supporters - crowdfunders - involved work closely with the entrepreneur in a cooperative way, participating actively in the development of the project initiated with feedbacks. The crowdfunding platform was noted as an intermediary that facilitates the repeated interaction between the proponent and supporters, creating not only financial value. It showed that the creation and exchange of new knowledge in the innovation phase generates non-financial benefits to the project, since it gains the possibility of adapting the value proposition to the market without the need for expensive research. It concludes by proposing future researches, such as: concentrating efforts to analyze the specific content worked on successful crowdfunding projects and understand how this detail can aid in reciprocity strategies in the context of social exchange and value co-creation.

Keywords: Social Exchange Theory. Value Co-creation. Crowdfunding. Collaborative Funding. Crowdfunders.

\section{Resumen}

El presente ensayo teórico investigó el fenómeno del crowdfunding a partir de la Teoría del Cambio Social y de la Co-creación de Valor con el propósito de establecer proposiciones de investigaciones futuras. Se percibió que crowdfunding presenta dinámicas de co-creación en sus procesos, donde los potenciales apoyadores - crowdfunders - involucrados trabajan en estrecha colaboración con el empresario de forma cooperativa, participando activamente en el desarrollo del proyecto instaurado con feedbacks. Se notó la plataforma crowdfunding como intermediario que facilita la interacción repetida entre el proponente y los partidarios, creando valor no sólo financiero. Se mostró que la creación e intercambio de nuevos conocimientos en la fase de innovación genera beneficios no financieros al proyecto, ya que gana la posibilidad de adaptar la propuesta de valor al mercado sin la necesidad de costosas investigaciones. Se concluye presentando proposiciones de futuras investigaciones, como: concentrar esfuerzos para analizar el contenido específico trabajado en proyectos exitosos de crowdfunding y comprender de qué manera ese detalle puede auxiliar en estrategias de reciprocidad en el contexto de intercambio social y co-creación de valor.

Palabras-clave: Teoría del Intercambio Social. Co-creación de Valor. Crowdfunding. Financiamiento Colaborativo. Crowdfunders.

\section{Introdução}

As duas últimas décadas têm sido dominadas por uma revolução tecnológica orientada a melhorar resultados, que transformou a sociedade em todos os seus aspectos, mais especificamente nos negócios empresariais e no marketing (CASTRO; SUAIDEN, 2015, KARJALUOTO; MUSTONEN; ULKUNIEMI, 2015, BALDO 2017). Um fenômeno desencadeado por essa transformação, segundo Hamari, Sjöklint e Ukkonen (2016), foi a economia compartilhada, que surgiu de uma série de desenvolvimentos tecnológicos que simplificaram o compartilhamento de produtos e serviços físicos e não-físicos por meio da disponibilidade de vários sistemas de informação na Internet. 
O progresso de sofisticadas tecnologias de web e a manifestação de sociedades online são os pilares de um novo sistema socioeconômico alternativo de produção (PIGATTO; QUEIROZ; LOURENZANI, 2015). Este desenvolvimento conduziu à mudança de paradigma que, para Mollick (2014), tem facilitado o surgimento de empresas e indivíduos ingressantes na plataforma de financiamento colaborativo conhecida como crowdfunding, modelo este que, para Botsman e Rogers (2011), faz parte da prática da economia compartilhada.

O crowdfunding é um método inovador para financiamento de novos negócios, permitindo fazer investimentos em projetos com ou sem fins lucrativos (BELLEFLAME; LAMBERT; SCHWIENBACHER, 2014), subsidiado por um grupo de indivíduos considerados potenciais consumidores (GRIFFIN, 2013). Ou seja, é uma comunidade cooperativa que corresponde à solicitação on-line para recursos de um público distribuído muitas vezes em troca de uma recompensa para quem apoia o projeto de negócio anunciado (GERBER; HUI, 2013). Na maioria dos projetos, o investimento só é realizado quando participam apoiadores suficientes e quando a soma total do capital para realização do projeto - e que é indicada antecipadamente - é alcançada.

Ao longo dos últimos dez anos, Mollick (2014) e Steffen (2015) afirmam que o fenômeno crowdfunding tem recebido bastante atenção no mundo e, conforme a Catarse (2014), também no Brasil, como um modelo de financiamento alternativo, trabalhado no ambiente on-line. Pesquisa divulgada pelo site Pequenas Empresas \& Grandes Negócios (2017) alega que mais de 50 mil empreendedores já conseguiram alavancar suas propostas de negócio a partir de recursos que arrecadaram com seus projetos inscritos na plataforma de crowdfunding chamada Kickante. Esta plataforma de financiamento colaborativo já recolheu 57 milhões em campanhas, aumentando seu faturamento em 30\% por ano, em relação ao ano anterior (FORBES, 2015). Para substanciar o realce do tema deste artigo, o site StartSe (2017) anunciou que mais de R \$ 180 milhões já foram transferidos no Brasil por meio de plataformas de crowdfunding, sendo um terço desse total apenas no ano de 2016. A investigação sobre o tema no Brasil é presente, e cada vez mais precisa de detalhes para a compreensão do seu funcionamento e impacto, como por exemplo, o estudo de Steffen (2014) ao descrever e analisar as plataformas brasileiras de crowdfunding.

Desta forma, percebe-se que a dinâmica de crowdfunding, troca entre projetos e possíveis interessados, alinha-se às palavras de Bagozzi (1995), que para satisfazer as necessidades humanas, as pessoas e organizações (com suas propostas) são compelidas a se engajar em trocas sociais e econômicas com outras pessoas e organizações. Dessa maneira, as trocas são a sustentação do conceito de marketing aparentemente trabalhado em crowdfunding.

Para Sheth, Gardner e Garret (1988), o marketing se faz presente onde existe atividades de troca. A colaboração ativa na troca entre produtores e consumidores, facilitada por uma empresa, para desenvolvimento de novos produtos ou serviços denota-se co-criação (PRAHALAD; RAMASWAMY, 2004a). Nesse sentido, o processo de lançamento de uma campanha de crowdfunding também é um processo de co-criação (NEVIN et al., 2017). 
Quando um projeto de crowdfunding é apresentado a uma multidão de patrocinadores potenciais, o resultado de se financiar ou não, de acordo com Guirado e Castro (2015), é um teste de mercado. Por isso, o desenvolvimento das interações sociais em torno de um determinado projeto e a natureza do próprio processo de desenvolvimento podem ser críticos para o resultado do esforço de crowdfunding (BURTCH; GHOSE; WATTAL, 2013).

A plataforma de crowdfunding se tornou um processo de co-criação, onde os patrocinadores trabalham em estreita colaboração com o empresário de forma cooperativa e participam ativamente do desenvolvimento do projeto crowdfunding, como testar protótipos iniciais e promover o projeto por meio do boca-a-boca (LEHNER, 2013). A maioria das plataformas de crowdfunding, incluindo a Catarse e Kickante, oferecem comunidades online para empresários e patrocinadores possam compartilhar ideias entre si, evidenciando um comportamento de co-criação (YI; GONG, 2013). O significado compartilhado entre empresários e patrocinadores pode desempenhar papéis importantes no processo de criação de valor. Para os empresários, crowdfunding é a abordagem para arrecadar dinheiro, anunciar produtos e serviços e obter feedback para melhorar os produtos e serviços (BELLEFLAME; LAMBERT; SCHWIENBACHER, 2014). Para investidores ou patrocinadores, a participação em projetos de crowdfunding pode resultar em maior retorno financeiro, reputação social e aproveitamento do sucesso das iniciativas de negócios (GERBER; HUI, 2013). Nesse ambiente, os apoiadores também são chamados de crowdfunders, que segundo Belleflame, Lambert e Schwienbacher (2014), não necessariamente se intitulam consumidores e não são meramente motivados por ganhos financeiros; eles também procuram benefícios sociais relacionados à atividade de troca, o que poderia ser a experiência no caso de crowdfunding com base em recompensas.

Uma das perspectivas para a prática de co-criação, de acordo com Fuller e Bilgram (2017), é a inovação colaborativa, que faz com que o consumidor possa participar com ideias para melhorar a performance de produtos e serviços. Nessa lógica, ao colaborar, esse apoiador passa a ser um co-criador para um produto e/ou serviço inovador, inclusive estimulando outras pessoas a realizarem subsequentes colaborações (BERNARDES; LUCIAN, 2015). Do mesmo modo, o crowdfunding envolve o consumidor de forma financeira e, ao fazêlo, conforme estudos feitos na Itália por Cucari e Nuhu (2017), este modelo maximiza o envolvimento dos consumidores no processo de co-criação de valor. Nesta lógica, o fenômeno do crowdfunding envolve usuários que se relacionam e co-criam diferentes tipos de valor, incluindo o valor econômico, mas não exclusivamente, mesmo quando as finanças parecem ser o principal objetivo (QUERO; VENTURA; KELLEHER, 2016).

Consoante a isso, Prahalad e Ramaswamy (2004a) difundiram o conceito de cocriação de valor alicerçado a uma perspectiva de lógica de serviço, em que o mercado é percebido como um fórum aberto em que o valor não é mais concebido unilateralmente por uma empresa e entregue ao consumidor, mas co-criado das interações entre negócios e clientes. Esta perspectiva é o quesito elementar da Lógica Dominante do Serviço em Marketing (SDL) que, de acordo com Vargo e Lusch (2008), os serviços e não 
impreterivelmente os produtos/bens devem ser a unidade elementar para a troca de valor. Para a dinâmica SDL, o sistema de serviço é uma configuração de recursos, incluindo pessoas, informações e tecnologia, conectados a outros sistemas por proposições de valor (VARGO; LUSCH, 2008). Assim, para Quero, Ventura e Kelleher (2016), entende-se que o crowdfunding também pode facilitar a co-criação de valor entre usuário sintonizados em uma plataforma e iniciativa de crowdfunding.

Depreende-se, assim, que o recurso de co-criação pode ser melhor compreendido pela aplicação da Teoria da Troca Social ou Social Exchange Theory (SET), pois fornece construções e conceitos adequados que podem auxiliar no conhecimento sobre as relações de troca durante os processos conjuntos entre empresa e consumidor, onde o cliente é concebido como um ativo na dinâmica criação de valor (PRAHALAD; RAMASWAMY, 2004a). A SET é uma teoria sociológica que analisou originalmente o processo de instituir e aprimorar trocas interpessoais (THIBAUT; KELLEY, 1959); com isso a SET preconiza que os participantes dessas relações de troca tendem a avaliar custos e benefícios, para que se comprometam com os projetos que lhes trazem vantagens sociais e econômicas (BRIGGS; GRISAFFE, 2010). Desse modo, para Homans (1958), quando os benefícios são bilaterais e mútuos as partes envolvidas geralmente decidem sustentar o relacionamento. Assim, a SET fornece uma sólida experiência em criação de valor, pois os participantes desses processos geralmente têm expectativas positivas e também percebem um ganho de status devido à sua cooperação no processo de desenvolvimento, levando-os a ter um compromisso com os projetos em que se envolvem (PREIKSCHAS et al., 2017).

Apesar das inúmeras pesquisas recentes que abordam a importância de se entender a interface entre as práticas de crowdfunding e marketing, nenhuma delas enfoca sobre uma interação mais transparente entre a teoria da troca social e co-criação nesse ambiente. Desse modo, o presente ensaio teórico se destina inicialmente a explorar conceitualmente a performance do crowdfunding entre os domínios do estudo da teoria da troca social e co-criação para, em seguida, por meio de proposições de pesquisa, apresentar ideias que refletem as relações existentes entre os mesmos, bem como as possíveis implicações das atividades de um ou de outro na motivação colaborativa. O objetivo, portanto, é refletir sobre um fenômeno que tem sido alvo de atenção dos pesquisadores, porém sob uma nova perspectiva, a fim de levantar novas questões para serem estudadas em pesquisas futuras.

\section{O Crowdfunding na perspectiva do Marketing}

O crowdfunding foi criado para permitir que pessoas interessadas em novidades e lançamentos de produtos e serviço ao mercado pudessem, com uma plataforma digital, captar o apoio direto de suas redes sociais e potencialmente de um público mais amplo para financiar os projetos inscritos na plataforma por meio de colaborações financeiras ou nãofinanceiras on-line. Contudo, o ambiente crowdfunding não apresenta simplesmente uma oportunidade de angariação de fundos, mas, também, o mais importante para a presente 
pesquisa, um grande potencial de expansão e incremento na compreensão dos esforços de marketing praticados nesse processo. Como predisposto por Ordanini et al. (2011), Mollick (2014) e Steffen (2015), uma campanha de crowdfunding permite medir o interesse público em um determinado produto ou serviço, como também noticia os serviços de captação de recursos on-line movimentados, ou seja, elucida-se aos potenciais futuros clientes que apoiam o projeto.

No contexto de marketing, orientar-se ao mercado significa trabalhar estratégias sólidas e fundamentadas, resultando no desempenho superior (DAMACENA; MARRA; PETROLL, 2012). Devido a isto, Steinberg e Demaria (2012) corroboram a relevância de se manter firme o esforço de marketing com o consumidor em negócios instaurados no crowdfunding, pois as pessoas só colaborarão se souberem como o projeto funciona. Vale ressaltar que as ações orientadas ao mercado ajudam a estruturar discussões sobre o futuro do consumo, onde estas, mediante a convergência digital, resultam em estímulos de mercado com uma participação mais ativa do consumidor (BERNARDES; LUCIAN, 2015).

Um estudo publicado por Carvalho e Rocha (2014) explora as ações promocionais de marketing utilizadas por artistas musicais para a comunicação de projetos difundidos em plataformas de crowdfunding. O resultado demonstra a eficiência dessas estratégias para a comunicação e relacionamento com o público-alvo, aliadas também à divulgação do projeto. Porém, para promover com êxito uma proposta de crowdfunding, é fundamental seguir estratégias concisas ao marketing, como semear a propagação do projeto usando esforços de marketing estabelecidos na dinâmica de lançamento de produto (MATTAR et al., 2013), assim, podendo-se almejar e converter visitantes da plataforma on-line em apoiadores.

Algumas funções de marketing em plataformas crowdfunding são: a pesquisa (LEHNER, 2013), a promoção (VOELKER; MCGLASHAN, 2013) e o engajamento (ORDANINI et al., 2011, MARIANI; CATALDO; VASTOLA, 2014). A função de pesquisa presume o crowdfunding como uma ferramenta de pesquisa de mercado (KOTLER; KELLER, 2012); afinal, uma campanha permite receber feedback sobre um determinado produto, bem como estimar a potencial base de clientes (SANTOS; BRASIL, 2010). Alguns projetos oferecem aos seus apoiadores a oportunidade de participar na concepção dos produtos/serviços (GRIFFIN, 2013). Para Monteiro (2014) e Bernardes e Lucian (2015), essa co-criação por parte dos consumidores muitas vezes permite um incremento estratégico ao bem, tornando-o mais atraente ao mercado potencial. Desse modo, trabalhar a interação com os usuários auxilia na ponderação da taxa de sucesso do futuro bem (BELLEFLAME; LAMBERT; SCHWIENBACHER, 2014), já que os consumidores proativos se tornam cada vez mais convergentes em um mercado onde o valor é criado por meio de sua relação (PRAHALAD; RAMASWAMY, 2004a).

Em conformidade com o contexto, Dong, Evan e Zou (2008) reconhecem que os consumidores estão comprometidos no processo de co-criação de valor e encorajá-los a participarem cada vez mais apoia a busca por vantagem competitiva. Por isso, para acompanhar essa revolução, os responsáveis pelo negócio devem almejar novas fontes de 
inovação, como a co-criação (PRAHALAD; RAMASWAMY, 2004b). Observa-se, então, que no processo de criação de valor convencional, empresas e consumidores tinham papéis modestos de produção e consumo, no entanto, essa distinção desaparece na co-criação.

Em vista disso, o conceito de marketing abarca constante discussão ao considerar fatores como a mudança de movimento no mercado e presença ativa do consumidor nos processos de criação de valor; por essa razão, a ideia clássica de troca presente nas definições de marketing (BAGOZZI, 1975), tem angariado a abordagem de troca acrescida de interações entre empresas e consumidores (GUMMESSON, 1987). Assim, equitativamente o conceito de valor na co-criação, tem chamado atenção na literatura de marketing (VARGO; LUSCH, 2004a). Perante isso, Vargo e Lusch (2004a), admitem que o marketing herdou um paradigma de troca da economia, cuja lógica dominante era alicerçada na troca de produtos manufaturados, estando em seu âmago, os recursos tangíveis, o valor agregado e as transações. Contudo, uma nova diretriz econômica vem se afirmando, com destaque aos recursos intangíveis e a co-criação de valor.

Enfim, o crowdfunding passa por não ignorar sua dimensão econômica como uma prática de consumo. Entretanto, e vale destacar, é uma prática contemporânea que funciona sob bases muito diferentes das tradicionais, principalmente na proposição de um processo na qual a co-criação é a base fundamental, bem como a diluição da dicotomia produtorconsumidor (STRAPASSON; NARDELLI; UENO, 2016).

\section{Teoria da Troca Social - Social Exchange Theory (SET)}

De acordo com Homans (1958), fundador da teoria da troca social, o comportamento social é uma troca de bens materiais e não materiais, durante os quais uma pessoa visa não apenas a maximização do lucro financeiro, mas também busca o estado de equilíbrio entre seus custos, insumos e rendimentos, como também os ganhos de outros participantes. Essa teoria propõe que, durante as interações sociais, os vários comportamentos sociais dos indivíduos são tipos de troca de bens materiais ou não materiais (HOMANS, 1958). Um dos princípios básicos dessa teoria é que os relacionamentos evoluem ao longo do tempo em relações confiantes, leais e mútuas. Para tal fim, os participantes precisam respeitar certas “regras” de troca. Segundo Emerson (1976), as regras de troca formam uma definição normativa da situação que se forma entre ou que é assumida pelas partes em uma relação de troca. Nesse sentido, as regras e normas de troca são as diretrizes dos processos de troca.

A reciprocidade é uma dessas normas sustentada pelo comportamento (social) humano. Por esse ângulo, Gouldner (1960) defende que as pessoas comprometem, umas às outras, deveres por causa de suas ações anteriores. A reciprocidade no contexto do crowdfunding acontece quando os projetos na plataforma se sentem obrigados a retribuir pelo dinheiro que receberam de um determinado apoiador. Esta obrigação é chamada de reciprocidade específica e é sustentada por sentimentos como "Eu lhe ajudo como você me ajudou no passado” (FARAJ; JOHNSON, 2011). Assim, os participantes de uma 
plataforma de crowdfunding podem se sentir obrigados a mostrar o seu apoio a outros projetos porque eles são gratos por terem recebido apoio no passado ou porque esperam precisar de suporte no futuro. Afinal, um ingrediente natural do crowdfunding é o da reciprocidade (WOLF, 2017).

A proposição é que as plataformas de crowdfunding baseadas em recompensas induzem fortes relações recíprocas entre seus usuários, que por sua vez desempenham um papel positivo no sucesso das campanhas. Percebe-se que uma característica sólida do crowdfunding é a reciprocidade entre o criador do projeto e o apoiador. Embora isso não precise ser uma troca, necessariamente, física, a reciprocidade é, muitas vezes, cimentada por meio da doação de fundos do patrocinador e alguma contribuição em resposta - produto físico, prazer compartilhado de saída, agradecimentos pessoais - do criador. Como evidencia a Figura 1, o projeto instaurado de uma cafeteira portátil que não se utiliza de energia elétrica ou cápsulas para fazer café expresso em qualquer lugar (CATARSE, 2016), arrecadou 723\% do que foi idealizado, usufruindo do modelo de recompensas como estratégia.

Figura 1 - Exemplos de recompensas promulgadas pelo projeto de crowdfunding
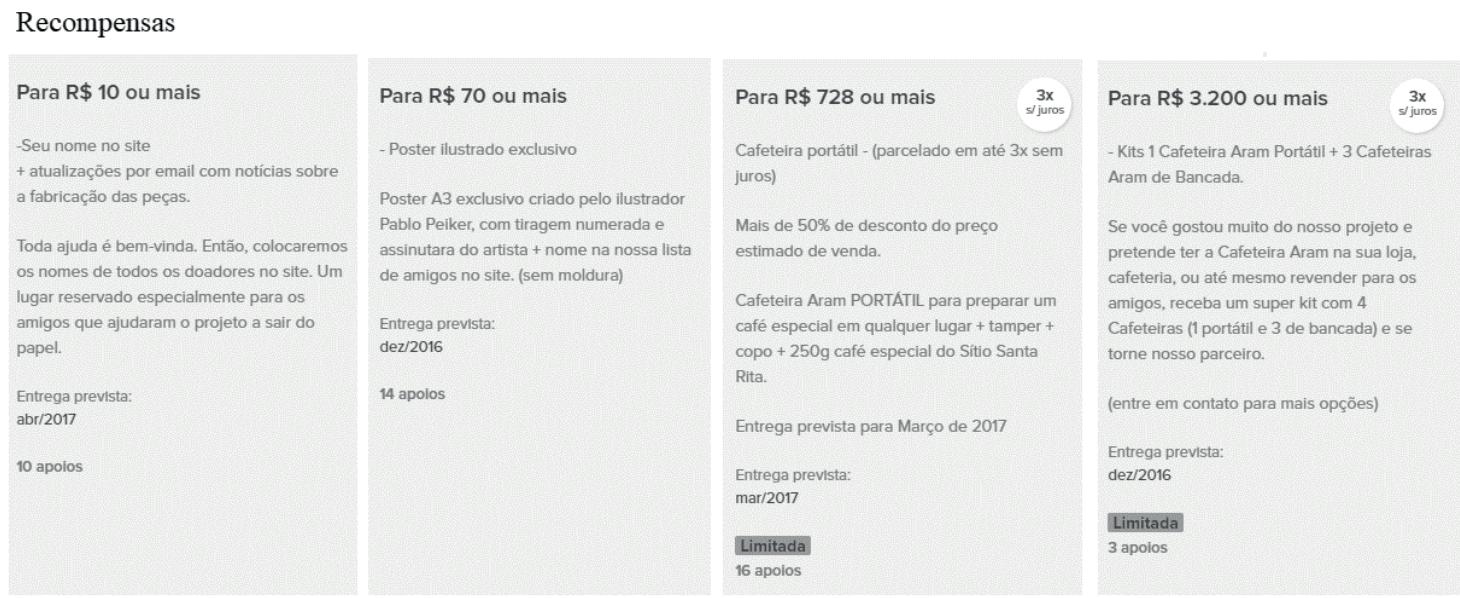

Fonte: Projeto Cafeteria Aram - Plataforma Catarse (2016).

Nota-se, em resposta a ações amigáveis ou justas, como oferecer uma recompensa simbólica ou personalizada, as plataformas de crowdfunding podem incitar os usuários a responder positivamente a um pedido para participação. Dessa maneira, a reciprocidade se mostra ser o núcleo das relações de marketing (BAGOZZI 1995).

Outro contexto evidente no ambiente de crowdfunding, anunciado por Kunz et al. (2017), é o efeito positivo entre a experiência de financiamento anterior de um projeto e a probabilidade de sucesso de sua própria campanha. Isto posto, sugere o estudo de Zvilichovsky, Yael e Barzilay (2015), os quais descobriram que os proponentes de projetos ativos, ao 
apoiarem outros projetos na plataforma, têm maiores taxas de sucesso atraindo, assim, mais apoiadores e coletando capital, que se torna possível em virtude de ações fundamentadas no princípio da reciprocidade.

Uma ideia idealizada na plataforma Kickstarter ajuda a compreender como a reciprocidade é evidente na prática de crowdfunding na iniciativa nomeada "Kicking It Forward” (KICKING IT FORWARD, 2017), plataforma onde os membros do Kickstarter constituem uma comunidade e são direcionados para se apoiarem mutuamente. Uma vez que o grau de compromisso em termos de apoios de projetos do proponente do projeto está disponível publicamente, os patrocinadores potenciais podem usar essas informações para firmar sua decisão de suporte. Caso um proponente esteja firmemente enraizado na comunidade Kickstarter, tal comportamento realça aos outros membros reconhecimento e compromisso e, assim, acontece a promoção do respectivo projeto.

Baseado nestas considerações, é coerente sugerir a seguinte proposição:

P1: Para o sucesso dos projetos de crowdfunding, torna-se importante trabalhar a reciprocidade em um contexto de troca social.

Consoante a P1, Moutinho e Leite (2013), ao realizarem uma análise econométrica sobre o desempenho das atividades na plataforma de crowdfunding Kickstarter, anunciaram a existência de fatores críticos para o sucesso de um projeto, sendo um deles a interação constante com os apoiadores. Nessa circunstância, Zvilichovsky, Yael e Barzilay (2013) investigaram mais de 75 mil projetos na Kickstarter e destacaram o efeito da reciprocidade nas práticas de interação proporcionadas pela campanha do projeto, afinal, segundo Faraj e Johnson (2011), a reciprocidade motiva contribuições. Os participantes que se envolvem em tais contribuições não necessariamente esperam receber ajuda futura dos mesmos indivíduos que auxiliaram, mas, em última instância, podem esperar receber apoio de outros na comunidade on-line. Percebe-se que este fenômeno de troca social com dinâmica de economia compartilhada não é apenas prevalente no contexto de plataformas de crowdfunding, mas que também tem um efeito significativo nos resultados; isto é, a vantagem pode ser acumulada em ambos os lados (projeto e apoiador). Sendo assim:

P2: Quanto maior for a utilização de estratégias de reciprocidade nos projetos de crowdfunding, maior será a troca social de possíveis apoiadores na plataforma.

Os indivíduos ativos em plataformas de crowdfunding podem se motivar a ajudar uma campanha específica com quem eles têm algum tipo de vínculo social, como aconteceu no projeto intitulado Bel Pesce: Legado A Menina do Vale - Figura 2 -, que arrecadou 342\% do investimento que necessitava (KICKANTE, 2017a); esses indivíduos desejam manter ou desenvolver um vínculo social e/ou são afetados pelos princípios da troca social (HOMANS, 1958). Assim, para os financiadores que não são financeiramente motivados, mas quem tem 
vínculos sociais, as interações provavelmente serão inspiradas pela dinâmica de influências sociais. Dessa maneira, o crowdfunding também fornece um teste de como a teoria da troca social se aplica neste contexto.

Figura 2 - Comentários estabelecidos em um projeto na plataforma crowdfunding

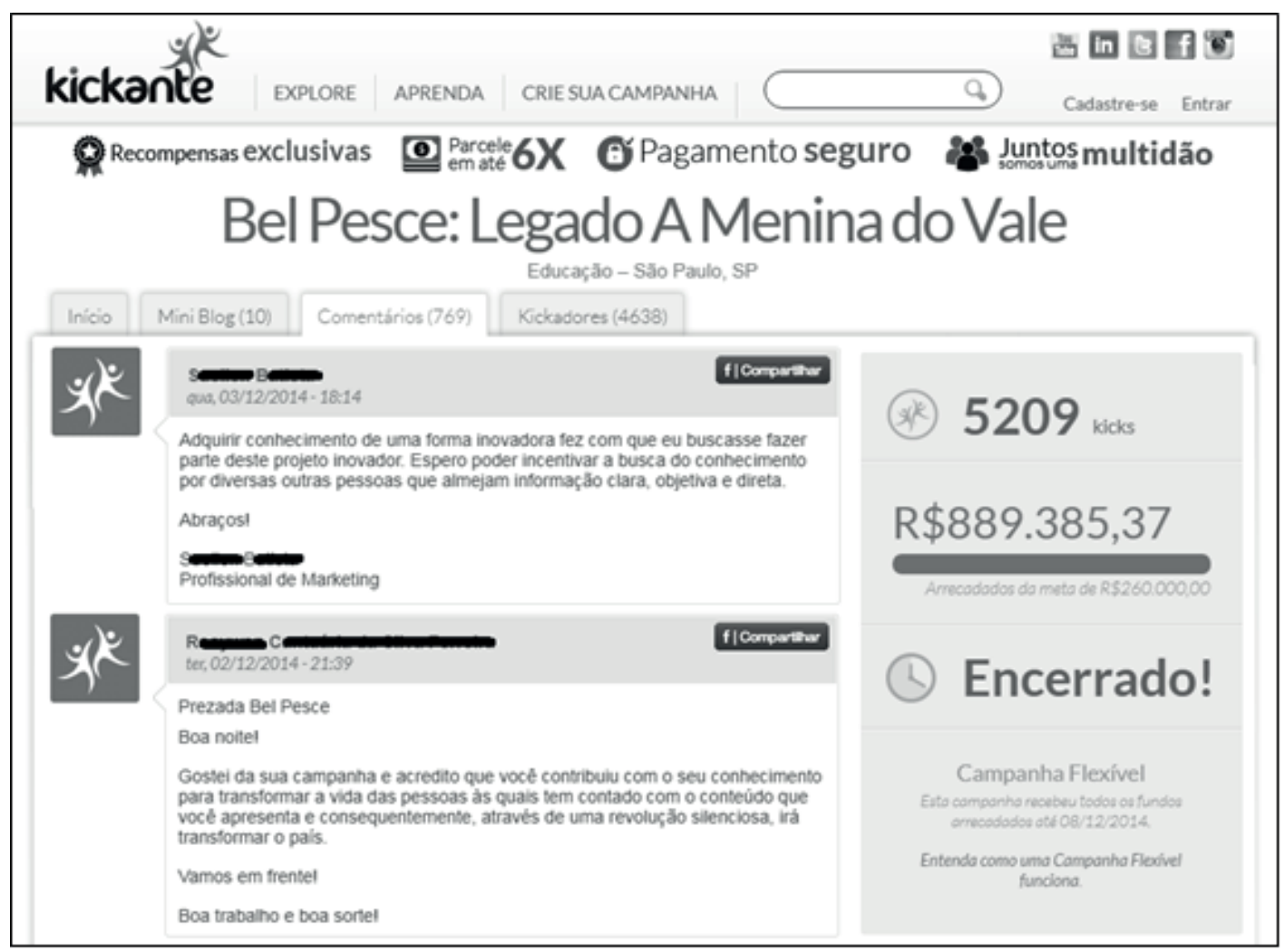

Fonte: Plataforma de crowdfunding Kickante (2017a).

A pesquisa sobre a teoria da troca social tem sido estudada no campo do marketing (BAGOZZI, 1974, BAGOZZI, 1975, MORGAN; HUNT, 1994). Nessa conjuntura, a troca apareceu como elemento central do conceito de marketing, como proposto pela American Marketing Association - AMA em 2013, “(...) é a atividade, conjunto de instituições e processos para criar, comunicar, entregar e trocar ofertas que tenham valor para consumidores, clientes, parceiros e a sociedade em geral” (Tradução nossa). Tal definição vai ao encontro do que foi promulgado por Bagozzi (1995): para satisfazer as necessidades humanas, as pessoas e organizações são compelidas a se engajar em trocas sociais e econômicas com outras pessoas e organizações.

Nota-se que existe nesta definição - a mais atual - da AMA de 2013, algo pontual que introduza uma orientação para o mercado, já que se preocupa com a troca de oferta de valor, com isso Grönroos (2013) propõe que marketing nasce de uma troca mútua e da 
manutenção de promessas ao estabelecer relações de longo prazo com os clientes, à medida que os objetivos das partes envolvidas sejam atendidos. No crowdfunding, os financiadores esperam receber uma recompensa do criador do projeto por seu apoio, lembrando que a troca de mercado é mais do que uma transferência de um produto ou serviço por dinheiro (BAGOZZI, 1975).

Em pesquisa desenvolvida sobre as motivações dos apoiadores/patrocinadores envolvidos em plataformas de crowdfunding, Ryu e Kim (2016) identificaram ser a recompensa uma das causas da estrutura motivacional, como também mostraram ser uma identidade dos tipos de apoiadores/patrocinadores que refletem a natureza do crowdfunding como uma nova forma de co-criação no contexto tecnológico.

Com base em dados analisados de uma plataforma de crowdfunding, Kunz et al. (2017) indicam que os laços sociais, inclusive, o fornecimento de recompensas influencia positivamente a probabilidade de sucesso de um projeto. Nesse sentido, na Figura 3, observase um exemplo de anunciação de recompensas.

Figura 3-Exemplo de recompensas estabelecidas em um projeto na plataforma crowdfunding

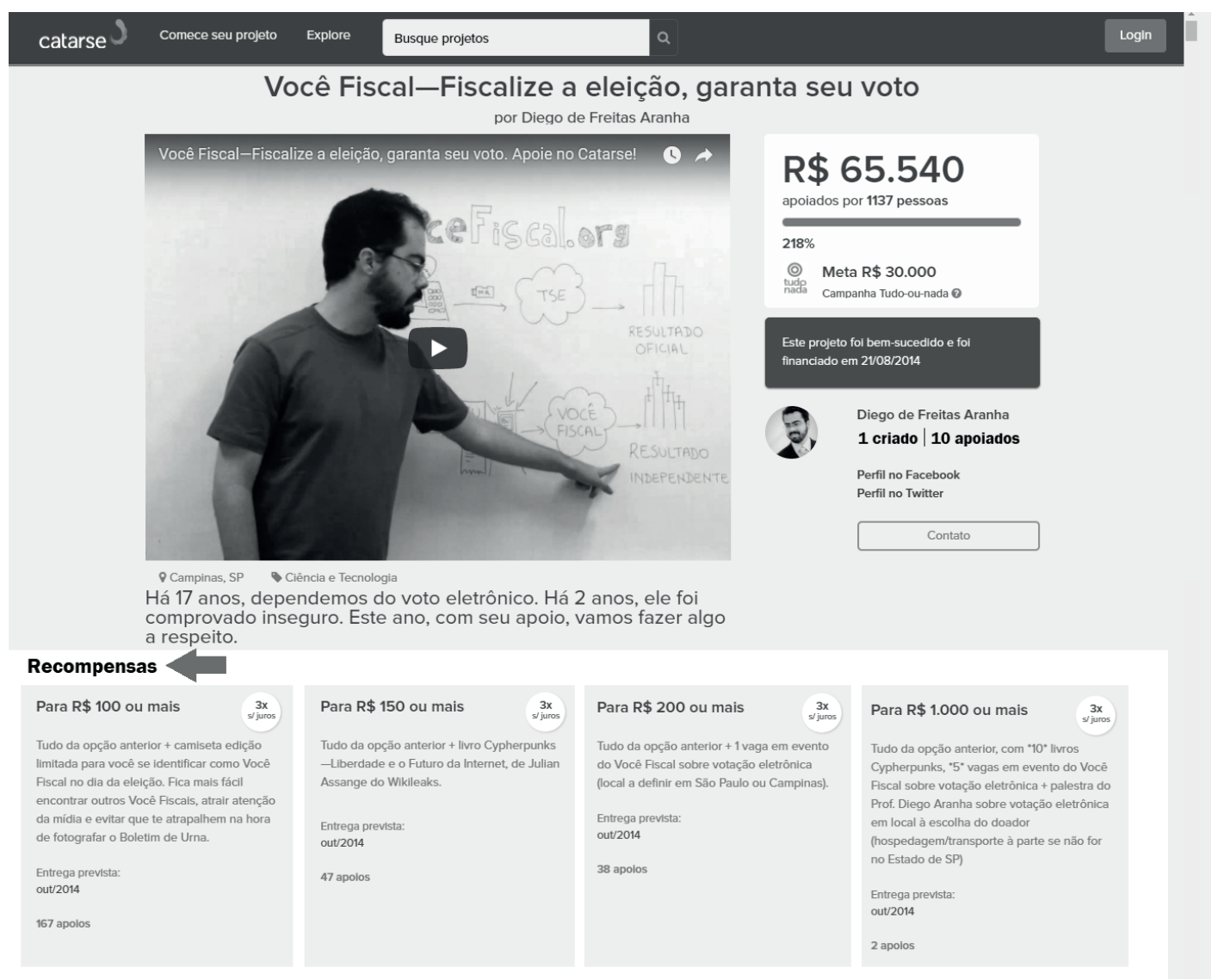

Fonte: Plataforma de crowdfunding Catarse (2017). 
Constata-se que um aspecto intrínseco ao modelo do crowdfunding são as recompensas, isto é, o que quem apoia o projeto na plataforma poderá receber em troca pela sua colaboração, sendo muitas vezes um item essencial aos financiadores (HOBBS; GRIGORE; MOLESWORTH, 2016). Estas recompensas poderão ser particulares ao próprio modelo da plataforma, como a partilha de receitas no caso das plataformas de investimento, ou fazer parte da estratégia de promoção do projeto, maximizando assim o valor levantado, por meio de contrapartidas apelativas do ponto de vista do financiador.

No crowdfunding, as recompensas tangíveis representam a troca utilitária que, segundo Bagozzi (1975, p. 36) é "uma interação pela qual os bens são dados em troca de dinheiro ou outros bens e a motivação por trás das ações reside no uso antecipado ou características tangíveis comumente associadas com os objetos na troca” (Tradução nossa). A troca simbólica está relacionada com recompensas intangíveis que se referem à "transferência mútua de entidades psicológicas, sociais ou outras entidades intangíveis entre duas ou mais partes” (Tradução nossa) (BAGOZZI, 1975, p. 36). Nota-se, também, que além de um presente como um sinal de gratidão, os doadores que fazem doações em plataformas crowdfunding geralmente não recebem nada em troca de sua doação. Isso significa que eles não adquirem a propriedade de risco, direitos de voto ou ações de lucro em troca de sua contribuição. O ato de doar em uma plataforma de crowdfunding, portanto, não pode ser visto como uma forma pura de troca econômica, onde os bens são oferecidos em troca de dinheiro ou outros bens (BAGOZZI, 1975). Nessa lógica, Cholakova e Clarysse (2015) revelaram que a decisão de participar do projeto crowdfunding é prevista pelo interesse dos indivíduos em receber recompensas. No entanto, muitas pessoas podem não ser apenas motivadas financeiramente, mas também intrinsecamente motivados.

Baseado nestas considerações, é coerente sugerir a seguinte proposição:

P3: Os crowdfunders (apoiadores/patrocinadores) são consumidores que estão procurando investir em um projeto e esperam recompensas materiais e imateriais.

Estreitamente ligado ao assunto das recompensas, é a questão da motivação dos crowdfunders (apoiadores/patrocinadores) em gerar quantidades maiores de seus rendimentos. Thurrild e Kamleitner (2016) enfatizam que os patrocinadores não são motivados necessariamente por recompensas materiais, mas, sim, predominantemente pelas recompensas imateriais e intrínsecos como, por exemplo, pela identificação pessoal com o tema do projeto e de seus objetivos.

Como as emoções positivas podem levar a um comportamento dirigido a metas (BAGOZZI; GOPINATH; NYER, 1999), pressupõe-se que o valor emocional desempenha um papel importante na intenção de conduzir a participação em um projeto de crowdfunding.

Baseado nestas considerações, é coerente sugerir a seguinte proposição: 
P4: O prazer em apoiar um projeto de crowdfunding tem um efeito positivo na intenção de investir em um projeto de crowdfunding.

A partir disso, declara-se que a teoria da troca social se manifesta na prática de crowdfunding, possivelmente na tomada de decisão; afinal, os seres humanos tentam minimizar as perdas e maximizar seus lucros durante o processo de troca (HOMANS, 1958). Um contexto contemporâneo de troca é o crowdfunding, método inovador para financiar uma variedade de novos empreendimentos, permitindo que fundadores individuais de projetos com fins lucrativos, não lucrativos, culturais ou sociais solicitem capital de muitos indivíduos, muitas vezes em troca de produtos futuros ou de equidade (MOLLICK, 2014). Nessa lógica, a teoria da troca social pode explicar a motivação do consumidor para o consumo nesse modelo de negócio.

\section{Co-criação de valor e a participação do potencial consumidor}

Várias abordagens teóricas têm sido pesquisadas para entender de que maneira a atividade desempenhada pelos consumidores pode ser válida e proveitosa para co-criar valor. Para Galvagno e Dalli (2014), em todas as abordagens, os consumidores são vistos como autores de atividades produtivas e, em cada uma, enfatizam-se diferentes atos que o consumidor desempenha para que o valor seja gerado com propósitos distintos para a co-criação.

A co-criação é considerada uma estratégia sólida para desenvolver com sucesso novos produtos estimados na era digital, ou seja, onde as plataformas de crowdfunding se estabelecem. Como efeito adicional, a pesquisa sobre a co-criação destacou que as experiências de co-criação podem estabelecer e intensificar relacionamentos benéficos para o consumidor (FULLER; BILGRAM, 2017). Para Vargo e Lusch (2004), os consumidores se envolvem em atividades virtuais de co-criação porque esperam que sua participação resulte em um sentimento prazeroso. Tal relação, é o resultado associado ao engajamento praticado, resultando possivelmente, em um melhor produto a ser ofertado ou recebimento de um incentivo do mercado, ou até, em uma tarefa ou o processo em que os consumidores desenvolvam valor. No contexto do crowdfunding, a co-criação de valor se dá quando alguém acredita e decide apoiar um determinado projeto. Ao colaborar, esse apoiador passa a ser um acreditador e co-criador de valor para um produto inovador, inclusive estimulando outras pessoas a realizarem subsequentes colaborações. Para esta forma comparativamente nova de "co-criação”, Fuller e Bilgram (2017) sugerem que uma experiência de co-criação agradável em um ambiente de crowdfunding pode aumentar o apoio da comunidade ao produto e aumentar a probabilidade de alcançar o objetivo de fomento. Na medida em que se aplica a co-criação, estreitam-se os laços, facilitando não apenas a troca de informações, 
mas também reduzindo as incertezas e possibilitando uma melhor assistência no pós-compra ao produto adquirido. A co-criação faz com que o cliente crie vínculos não apenas naquele produto com o qual colaborou, mas também naquela empresa que lhe deu essa oportunidade de participação (BERNARDES; LUCIAN, 2015).

Baseado nestas considerações, é coerente sugerir a seguinte proposição:

P5: Com a utilização de estratégias de co-criação de valor em plataformas de crowdfunding, o consumidor (usuário) que participa da concepção do bem que deseja fomentar, faz com que esse projeto (produto) seja percebido pelos consumidores com potencial satisfação de mercado.

Uma plataforma de crowdfuding, como a Catarse e a Kickante, fornece áreas de conversa on-line em que tanto os proponentes de projetos como os patrocinadores destes podem compartilhar informações. Os empresários inclusive são capazes de testar seus produtos e promovê-los, enquanto os potenciais apoiadores trabalham feedback social (BELLEFLAME; LAMBERT; SCHWIENBACHER, 2014). Desta forma, desenvolve-se um comportamento de co-criação (YI; GONG, 2013), que pode ser promovido por meio do desenvolvimento de uma descrição clara do projeto para compartilhar com os usuários de forma coletiva. Por exemplo, em uma plataforma de crowdfunding como a Catarse, os empresários conseguem descrever suas campanhas usando palavras, imagens, vídeos e anexando arquivos. Isso ressalta a importância estratégica, para um projeto de crowdfunding, para operar por meio de uma plataforma de tecnologia da informação que permita uma comunicação de informações imediata, completa e relacionada à interação.

Os financiadores, considerados apoiadores ou crowdfunders, contribuem para o projeto do empreendedor, não só com recursos monetários, mas também com ideias e informações. Isso é feito por meio de plataformas tecnológicas, que fornecem uma comunidade e as ferramentas para interagir com ele, como visto na Figura 4, que destaca o projeto intitulado Malalai, desenvolvedor de tecnologia para segurança pessoal (KICKANTE, 2017b). A proposta ingressou a plataforma de crowdfunding Kickante e obteve a contribuição necessária para conquistar a meta delimitada. 
Figura 4 - Comentários feitos pelos apoiadores do projeto Malalai

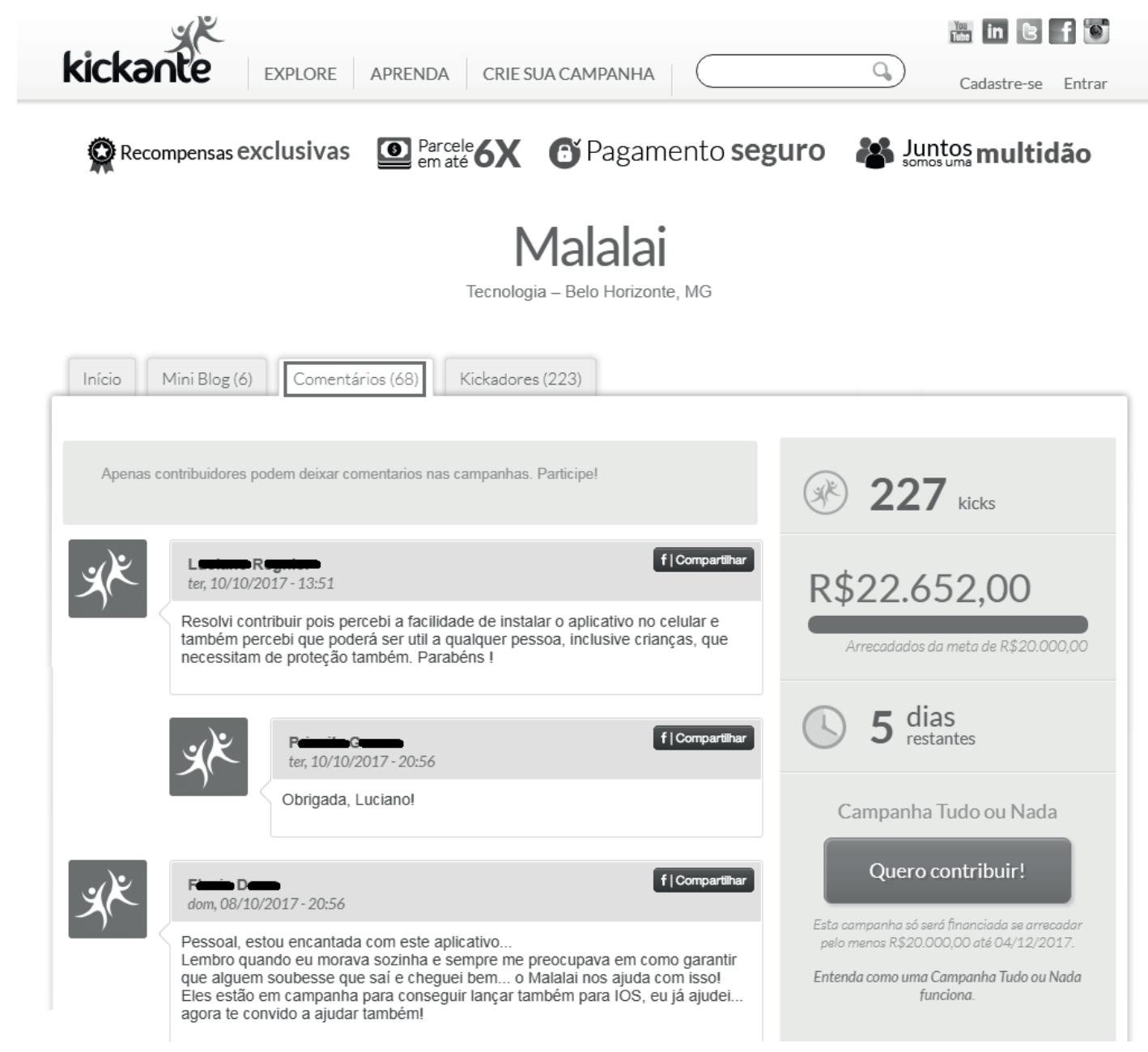

Fonte: Plataforma de crowdfunding Kickante (2017b).

A maioria das plataformas de crowdfunding fornece uma ferramenta de bate-papo onde ocorre um diálogo entre o empreendedor e seus potenciais financiadores (ZHENG; LI; XU, 2014). Os financiadores publicam seus comentários sobre o projeto e eles são respondidos ou refutados pelo empreendedor.

O sistema de crowdfunding é complexo no sentido descrito por Gummesson (2006): nele há agentes diferentes participando, e sua estrutura e funcionamento incluem funções muito mais amplas do que um mero financiamento, já que são estruturas criadas para permitir a co-criação de valor para todos os diferentes usuários por meio da aplicação dos recursos de todos os participantes, a fim de criar um produto orientado para o mercado e baseado em relacionamento. Portanto, Belleflamme, Lambert e Schwienbacher (2014) atestam que 
o crowdfunding compreende elementos de co-criação, sobretudo, por exemplo, quando os indivíduos fornecem feedback e ideias de preferências sobre o produto e desempenham um papel importante na promoção e comercialização do mesmo.

Prahalad e Ramaswamy (2004a) concentram sua teoria na importância de construir canais eficientes de encontro entre a empresa e os clientes, permitindo a realização de uma co-criação. Isso inclui a possibilidade de ter uma comunicação bidirecional com o cliente, integrá-los de acordo com suas competências no desenvolvimento do produto/serviço, compartilhar informações significativas e, consequentemente, organizar uma comunidade de marcas estruturada. Esses procedimentos são essenciais para criar valor que, de acordo com a teoria da lógica dominante do serviço, não pode ser produzido de forma autônoma pela empresa e depois entregue ao cliente, mas é a colaboração do cliente que dá valor ao produto e ao serviço (VARGO; LUSCH, 2008).

Portanto, durante uma campanha de crowdfunding, a empresa possui várias ferramentas para implementar esta teoria graças à funcionalidade das plataformas. No entanto, as literaturas de marketing e ciências sociais focaram principalmente no crowdfunding com o objetivo de analisar o crescimento impressionante das plataformas utilizadas pelas empresas como recurso financeiro alternativo (BELLEFLAME; LAMBERT; SCHWIENBACHER, 2014). Nesta óptica, o precedente vem de Ordanini et al. (2011), que se concentra nas razões pelas quais um consumidor deve se transformar em co-criador durante uma campanha de crowdfunding. Os autores demonstraram a importância da eficiência da rede para atrair co-criadores, mencionando que as empresas que organizam iniciativas desse tipo criam sistemas e condições organizacionais necessários para a integração de recursos entre os usuários (ORDANINI et al., 2011).

\section{Considerações finais}

O ambiente de negócios está se alterando pela sociedade, entrando em uma era digitalizada onde ideias e insights são abundantes, mais facilmente acessíveis e, portanto, atuam como co-criadores. Por isso, esta pesquisa é um primeiro passo para estudar a diversidade das relações interpessoais dentro de um conjunto mais amplo de práticas de crowdfunding, que mostra a gradação de expectativas e comportamentos nos sistemas de troca. Nesse sentido, o presente ensaio teórico investigou o fenômeno do crowdfunding entre as atribuições da ciência da Teoria da Troca Social e da Co-criação para poder apontar proposições que retratam as correlações existentes entre os mesmos. Portanto, o trabalho refletiu sobre um evento que tem sido muito pesquisado, mas sob um diferente aspecto, que despertou novas indagações a serem estudadas em pesquisas futuras.

A teoria da troca social serviu bem ao marketing por 40 anos, mas percebe-se que está dando lugar a conceitos relacionais, que estão modificando aspectos dos processos de marketing tradicionais. Como sugerido pelo ensaio, o contexto em que o valor foi criado mudou, 
aumentou em complexidade e o tipo de análise precisa acompanhar essa nova realidade. Nesse sentido, o contexto torna-se mais importante como um quadro de troca e experiência.

Assim, evidencia-se o crowdfunding como uma abordagem colaborativa de troca, que pode ser vista como uma atividade de co-criação, onde as pessoas podem participar da seleção e desenvolvimento dos projetos por meio do fornecimento de fundos ou feedbacks.

Em vista disso, este estudo apoiou a importância do uso da “apoiadores” para que as empresas obtenham ideias, comentários e conscientização sobre projetos (GUIRADO; CASTRO, 2015), para então, inspirar o desenvolvimento de novos produtos e/ou serviços ou melhorá-los (PRAHALAD; RAMASWAMY, 2004a). Um dos destaques do ambiente crowdfunding é poder envolver patrocinadores em todo o processo de implementação do projeto, participando ativamente do desenvolvimento do produto (LEHNER, 2013). Por isso, o crowdfunding pode ser considerado um tipo de co-criação de valor por apoiadores. Como percebido, o comportamento de co-criação está relacionado ao valor do cliente e à satisfação com o serviço. Nota-se, pelos exemplos identificados, que os participantes que estão ativamente envolvidos nas atividades de co-criação de valor vivem estímulos que os entusiasma mais pelo processo. Diante disso, vale sugerir uma pesquisa aprofundada para compreender em detalhes, a quarta proposição: O prazer em apoiar um projeto de crowdfunding tem um efeito positivo na intenção de investir em um projeto de crowdfunding, ou seja, como e quais são as atividades praticadas pelos apoiadores em campanhas crowdfunding que podem melhorar sua experiência e aumentar sua satisfação em projetos.

De fato, há muitas incógnitas sobre como a co-criação pode ser promovida, gerenciada e explorada pelas empresas e como um modelo de negócios pode ser projetado (ou reinventado) para se beneficiar da co-criação de valor. Nessa lógica, o fenômeno do crowdfunding é apenas uma fórmula entre muitos que, habilitado pelas novas tecnologias e pela Internet, proporcionou um ambiente adequado para co-criação de determinados projetos. No entanto, o desenvolvimento contínuo da tecnologia e as inter-relações crescentes entre diversos atores, como habilitado pela tecnologia, continuarão a facilitar o desenvolvimento de novos modelos, o que será interessante considerar tanto do ponto de vista teórico como empírico. Assim, conclui-se que a plataforma como intermediário facilita a interação repetida entre o proponente e apoiadores e que isso criará valor não só financeiro por meio da interação. Além disso, considera-se que a criação e troca de novos conhecimentos na fase de inovação gera benefícios não financeiros ao projeto, uma vez que ganha a possibilidade de adaptar sua proposta de valor ao mercado.

Pesquisas futuras podem validar por meio de experimentos bem planejados se as proposições deste ensaio são válidas, como: concentrar esforços para analisar o conteúdo específico trabalhado na apresentação de projetos bem-sucedidos em plataformas crowdfunding e compreender de que maneira esse detalhe pode auxiliar em estratégias de reciprocidade no contexto de troca social e co-criação de valor. Ao final, este ensaio ofereceu apenas uma discussão indutiva e informações preliminares sobre o fenômeno abordado 
e o novo papel do consumidor/apoiador/patrocinador, dessa maneira, pode-se ampliar o conhecimento de forma quantitativa, investigando a co-criação como propulsor do sucesso das atividades de financiamento colaborativo. Para tal, utilizar uma plataforma específica de crowdfunding como contexto de estudo para analisar e comparar as iniciativas financiadas versus não financiadas, de modo a identificar fatores pontuais do êxito.

A limitação intrínseca do estudo está ligada à análise e atribuição dos exemplos, que por sua vez foi instaurada por atividades brasileiras, como também plataformas nacionais. É provável que haja diferenças culturais, e até diferenças geracionais, sobre como o valor é percebido pelos empresários em outras nacionalidades. Com essas limitações em mente, seria interessante investigar o valor percebido pelos proponentes em distintas culturas, e se o contexto de crowdfunding pode ser percebido de forma diferente ao longo do tempo.

\section{Referências}

ALLIEDCROWDS CROWDFUNDING REPORT. Relatório Annual - Developing World Crowdfunding. 2016. Disponível em: https://www.crowdfundinsider.com/2016/01/80216-alliedcrowds-releases-january2016-crowdfunding-report/. Acesso em: 20 set. 2017.

AMERICAN MARKETING ASSOCIATION. Definition of Marketing. 2007. Disponível em: https://www. ama.org/AboutAMA/Pages/Definition-of-Marketing.aspx. Acesso em: 10 set. 2017.

BALDO, W. C. Cibercultura, automação e big data: questões sobre a evolução tecnológica e suas consequências para a comunicação e a sociedade. In: XXII CONGRESSO DE CIÊNCIAS DA COMUNICAÇÃO NA REGIÃO SUDESTE, 2014, Volta Redonda, RJ. Anais... Volta Redonda, RJ - Intercom - Sociedade Brasileira de Estudos Interdisciplinares da Comunicação. 2017.

BAGOZZI, R. P. Marketing as an organized behavioral system of exchange. Journal of Marketing, n. 38, p. 77-81, 1974.

BAGOZZI, R. P. Marketing as exchange. Journal of Marketing, v. 4, n. 39, p. 32-39, 1975.

BAGOZZI, R. P.; GOPINATH, M.; NYER, P. U. The role of emotions in marketing, Journal of the Academy of Marketing Science, v. 27, n. 2, p. 184-206, 1999.

BELLEFLAMME, P.; LAMBERT, T.; SCHWIENBACHER, A. Crowdfunding: tapping the right crowd. Journal of Business Venturing, v. 29, n. 5, p. 585-609, 2014.

BERNARDES, B.; LUCIAN, R. Crowdfunding: A Influência da Co-Criação e do Sentimento de Pertença na Satisfação dos Apoiadores Luso-Brasileiros. GESTÃO. Org-Revista Eletrônica de Gestão Organizacional, v. 13 (Ed. Especial), p. 360-369, 2015.

BOTSMAN, R.; ROGERS, R. O que é meu é seu: como o consumo coletivo está mudando o nosso mundo. Porto Alegre: Bookman, 2011.

BRIGGS, E; GRISAFFE, D. Service Performance-Loyalty Intentions Link in a Business-to-Business Context: the role of relational exchange outcomes and customer characteristics, Journal of Service Research, v. 13, n. 1, p. 37-51, 2010.

BURTCH, G.; GHOSE, A.; WATTAL, S. An empirical examination of the antecedents and consequences of contribution patterns in crowd-funded markets. Information Systems Research, v. 24, n. 3, p. 499519, 2013. 
CARVALHO, A. C. M. de; ROCHA, D. de C. Ações promocionais de marketing na divulgação de projetos musicais do crowdfunding. In: XXXVII CONGRESSO BRASILEIRO DE CIÊNCIAS DA COMUNICAÇÃO, 2014, Foz do Iguaçu, PR. Anais... Foz do Iguaçu - Intercom - Sociedade Brasileira de Estudos Interdisciplinares da Comunicação, 2014.

CHOLAKOVA, M.; CLARYSSE, B. Does the Possibility to Make Equity Investments in Crowdfunding Projects Crowd Out Reward-Based Investments?. Entrepreneurship Theory and Practice, v. 39, n. 1, p. 145-172, 2015.

CASTRO, M. DE F. C.; SUAIDEN, E. J. Fatores críticos de sucesso na oferta de produtos e serviços de informação na web. Pesquisa Brasileira em Ciência da Informação e Biblioteconomia, João Pessoa, v. 10, n. 1, p. 1-28, 2015.

CATARSE. Metas peludas e inspiradoras para 2014 e o futuro. 2014. Disponível em: http://blog.catarse. me/metas-peludas-e-inspiradoras-para-2014-e-para-o-futuro/. Acesso em: 20 jul. 2017.

CATARSE. Cafeteria Aram. 2016. Disponível em: https://www.catarse.me/cafeteiraaram?ref=ctrse_ explore\&project_id=43002. Acesso em: 20 set. 2017.

DAMACENA, C.; MARRA, G. dos S.; PETROLL, M. de la M. Orientação ao mercado, inovação colaborativa com os consumidores e sobrecarga de informação. Perspectivas Contemporâneas, Campo Mourão, v. 7, n. 2, p. 177-206, 2012.

DONG, B.; EVANS, K. R.; ZOU, S. J. The effects of customer participation in co-created service recovery. Journal of the Academy of Marketing Science. v. 36, n. 1, p. 123-137, 2008.

EMERSON, R. M. Social exchange theory. Annual Review of Sociology, v. 2, n. 1, p. 335-362, 1976.

FARAJ, S.; JOHNSON, S. L. Network exchange patterns in on-line communities. Organization Science, v. 22, n. 6, p. 1464-1480, 2011.

FORBES. The Indiegogo of Brazil: Candice Pascoal and Kickante. 2015. Disponível em: https://www.forbes. com/sites/joshsteimle/2015/09/03/the-indiegogo-of-brazil-candice-pascoal-and-kickante/\#7eb6cc3d6aeb. Acesso em: 10 set. 2017.

FULLER, J.; BILGRAM, V. The moderating effect of personal features on the consequences of an enjoyable co-creation experience. Journal of Product \& Brand Management, v. 26, n. 4, p. 386-401, 2017.

GALVAGNO, M.; DALLI, D. Theory of value co-creation: a systematic literature review. Managing Service Quality, v. 24, n. 6, p. 643-683, 2014.

GERBER, E. M.; HUI, J. S. Crowdfunding: Motivations and Deterrents for Participation. ACM Transactions on Computer-Human Interaction (TOCHI), v. 20, n. 6, p. 1-37, 2013.

GOULDNER, A. W. The norm of reciprocity: a preliminary statement. American Sociological Review, v. 25, n. 2, p. 161-178, 1960.

GRIFFIN, Z. Crowdfunding: fleecing the American masses. Journal of Law, Technology \& the Internet, v. 4, n. 2, p. 375-421, 2013.

GRÖNROOS, C. Relationship Marketing as Promise Management. In: MACLARAN, P.; SAREN, M.; STERN, B.; TADAJEWSKI, M. The SAGE Handbook of Marketing Theory. SAGE Publications: Thousand Oaks, 2013.

GUIRADO, C. E.; CASTRO, C. G. Crowdfunding as an Open Innovation for Co-Creation. In: ASSADI, D. (Ed.). Strategic Approaches to Successful Crowdfunding. Burgundy School of Business, France, p. 150174, 2015. 
GUMMESSON, E. The New Marketing: developing long-term interactive relationships. Long Range Planning, v. 20, n. 4, p. 10-20, 1987.

GUMMESSON, E. Many to many marketing as grand theory: a Nordic School contribution. In: LUSH, F.; VARGO, S. L. The Service - Dominant Logic of Marketing: Dialog, Debate and Directions. New York: ME Sharpe, 2006.

HAMARI, J.; SJÖKLINT, M.; UKKONEN, A. The sharing economy: Why people participate in collaborative consumption. Journal of the Association for Information Science and Technology, v. 67, n. 9, p. 20472059, 2016.

HOBBS, J.; GRIGORE, G.; MOLESWORTH, M. Success in the management of crowdfunding projects in the creative industries, Internet Research, v. 26, n. 1, p. 146-166, 2016.

HOMANS, G. C. Social behavior as exchange. American Journal of Sociology, v. 63, n. 6, p. 597-606, 1958. KARJALUOTO, H.; MUSTONEN, N.; ULKUNIEMI, P. The role of digital channels in industrial marketing communications, Journal of Business \& Industrial Marketing, v. 30, n. 6, p. 703-710, 2015.

KICKANTE. Campanha Bel Pesce: Legado A Menina do Vale. 2017. Disponível em: https://www.kickante. com.br/campanhas/bel-pesce-legado-menina-do-vale. Acesso em: 18 nov. 2017a.

KICKANTE. Campanha Malalai. 2017. Disponível em: https://www.kickante.com.br/campanhas/malalai. Acesso em: 18 nov. 2017b.

KICKING IT FORWARD. Frequently Asked Questions. 2017. Disponível em: https://kickingitforward. org/faq. Acesso em: 18 set. 2017.

KOTLER, P.; KELLER, K. L. Administração de marketing. 14. ed. São Paulo: Pearson Prentice Hall, 2012.

KUNZ, M. M.; BRETSCHNEIDER, U.; ERLER, M.; LEIMEISTER, J. M. An empirical investigation of signaling in reward-based crowdfunding. Electronic Commerce Research, v. 17, n. 3, p. 425-461, 2017.

LEHNER, O. M. Crowdfunding social ventures: a model and research agenda. Venture Capital, v. 15, n. 4, p. 289-311, 2013.

MARIANI, A.; CATALDO, A.; VASTOLA, A. Consumers' engagement in co-creation of value and Crowdfunding: Naked Wine as a Best Practice. Calitatea, v. 15, n. 143, p. 70-73, 2014.

MATTAR, F. N.; OLIVEIRA, B.; MORAES, C. A. de; QUEIROZ, M. J. de; QUEIROZ, R. S. B. De; MOTTA, S. L. S. Gerência de produtos: estratégias e ações para o sucesso. 1. ed. Rio de Janeiro: Elsevier Campus, 2013. MOLLICK, E. R. The dynamics of crowdfunding: an exploratory study. Journal of Business Venturing, v. 29, n. 1, p. 1-16, 2014.

MONTEIRO, M. de C. P. Crowdfunding no Brasil: uma análise sobre as motivações de quem participa. 2014. 209 f. Dissertação (Mestrado em Administração) - Programa de Pós-Graduação em Administração, Escola Brasileira de Administração Pública e de Empresas, Centro de Formação Acadêmica e Pesquisa da Fundação Getúlio do Rio de Janeiro, Rio de Janeiro, 2014.

MORGAN, R. M.; HUNT, S. D. The Commitment-Trust Theory of Relationship Marketing. Journal of Marketing, v. 58, p. 20-38, 1994.

MOUTINHO, N.; LEITE, P. V. Critical success factors in crowdfunding: the case of Kickstarter. Cad. do Merc. De. Valores Mobiliários, v. 45, p. 8-32, 2013.

NEVIN, S.; GLEASURE, R.; O’REILLY, P.; FELLER, J.; LI, S.; CRISTOFORO, J. Social Identity and Social Media Activities in Equity Crowdfunding. In: 13TH INTERNATIONAL SYMPOSIUM ON OPEN COLLABORATION. ACM, 2017. Proceedings... 
ORDANINI, A.; MICELI, L.; PIZZETTI, M.; PARASURAMAN, A. Crowd-funding: transforming customers into investors through innovative service platforms, Journal of Service Management, v. 22, n. 4, p. 443-470, 2011.

PEQUENAS EMPRESAS \& GRANDES NEGÓCIOS. Empreendedora cria plataforma de crowdfunding e arrecada R\$ 57 milhões em campanhas. 2017. Disponível em: http://revistapegn.globo.com/Mulheresempreendedoras/noticia/2017/09/empreendedora-cria-plataforma-de-crowdfunding-e-arrecada-r-57milhoes-em-campanhas.html. Acesso em: 10 set. 2017.

PIGATTO, G. A. S.; QUEIROZ, T. R.; LOURENZANI, A. E. B. S. Redes sociais de produtores de mandioca em regiões do estado de São Paulo. Interações (Campo Grande), Campo Grande, v. 16, n. 1, p. 75-86, 2015.

PRAHALAD, C. K.; RAMASWAMY, V. Co-creation experiences: the next practice in value creation. Journal of Interactive Marketing, v. 18, n. 3, p. 5-14, $2004 \mathrm{a}$.

PRAHALAD, C. K.; RAMASWAMY, V. The Future of Competition: Co-Creating Unique Value with Customers. Boston: Harvard Business School Press, 2004b.

PREIKSCHAS, M. W.; CABANELAS, P.; RÜDIGER, K.; LAMPÓN, J. F. Value co-creation, dynamic capabilities and customer retention in industrial markets. Journal of Business \& Industrial Marketing, v. 32, n. 3, p. 409-420, 2017.

QUERO, M. J.; VENTURA, R.; KELLEHER, C. Value-in-context in crowdfunding ecosystems: How context frames value co-creation. Service Business, v. 11, n. 2, p. 405-425, 2016.

RYU, S.; KIM, Y-G. A typology of crowdfunding sponsors: Birds of a feather flock together? Electronic Commerce Research and Applications, v. 16, p. 43-54, 2016.

SANTOS, C. R. dos; BRASIL, V. S. Envolvimento do consumidor em processos de desenvolvimento de produtos: um estudo qualitativo junto a empresas de bens de consumo. Revista de Administração de Empresas - RAE, v. 50, n. 3, p. 300-311, 2010.

SHETH, J.; GARDNER, D.; GARRET, D. Marketing theory: evolution and evaluation. New York: John Wiley \& Sons, 1988.

STARTSE. Crowdfunding se consolida com mais de R\$ 180 milhões em doações. 2017. Disponível em: https://conteudo.startse.com.br/startups/mariana-rodrigues/crowdfunding-se-consolida-com-mais-de-r-180milhoes-em-doacoes/. Acesso em: 10 set. 2017.

STEINBERG, S.; DEMARIA, R. The crowdfunding bible: how to raise money for any startup, video game or project. Kindle Edition: Read.Me, 2012.

STEFFEN, C. O Crowdfunding no Brasil de 2014. In: XXXVII CONGRESSO BRASILEIRO DE CIÊNCIAS DA COMUNICAÇÃO, 2014, Foz do Iguaçu, PR. Anais... Foz do Iguaçu - Intercom - Sociedade Brasileira de Estudos Interdisciplinares da Comunicação, 2014.

STEFFEN, C. Meios digitais participativos e economia criativa: uma exploração das plataformas Brasileiras de crowdfunding. Intexto, Porto Alegre, UFRGS, v. 32, p. 156-171, 2015.

STRAPASSON, E. V. L.; NARDELLI, M. A.; UENO, G. Crowdfunding no turismo comunitário: uma discussão conceitual. In: X FÓRUM INTERNACIONAL DE TURISMO DO IGUASSU, 2016, Foz do Iguaçu, PR. Anais... Foz do Iguaçu - Fórum Internacional de Turismo Do Iguassu, 2016.

THIBAUT, J. W.; KELLEY, H. H. The Social Psychology of Groups, New York, 1959.

THURRILD, C.; KAMLEITNER, B. What goes around comes around? California Management Review, v. 58, n. 2, p. 88-110, 2016. 
VARGO, S. L.; LUSCH, R. F. Evolving to a new dominant logic for marketing, Journal of Marketing, v. 68, n. 1, p. 1-17, 2004.

VARGO, S. L.; LUSCH, R. F. Service-dominant logic: continuing the evolution. Journal of the Academy of Marketing Science, v. 36, n.1, p. 1-10, 2008.

VARGO, S. L. Market Systems, stakeholders and value propositions. Towards a service-dominant logicbased theory of the market, European Journal of Marketing, v. 45, n. 1/2, p. 217-222, 2011.

VOELKER, T. A.; MCGLASHAN, R. What is Crowdfunding? Bringing the Power of Kickstarter to Your Entrepreneurship Research and Teaching Activities. Small Business Institute ${ }^{\circledR}$ Journal, v. 9, n. 2, p. 1122, 2013.

YI, Y.; GONG, T. Customer value co-creation behavior: scale development and validation. Journal of Business Research. v. 66, n. 9, p. 1279-1284, 2013.

WOLF, C. From Harambee to Modern Crowdfunding: the Opportunities and Challenges in Sub-Saharan Africa. In: REDFORD, D. T. (Ed.) Developing Africa’s Financial Services, p. 263-277, 2017.

ZHENG, H.; LI, D.; XU, Y. The role of multidimensional social capital in crowdfunding: A comparative study in Chine and US. Information \& Management, 51, 488-496, 2014.

ZVILICHOVSKY, D.; YAEL, I.; BARZILAY, O. Playing both sides of the market: Success and reciprocity on crowdfunding platforms. In: 34TH INTERNATIONAL CONFERENCE ON INFORMATION SYSTEMs (ICIS 2013). Milan, Italy, 2013. Proceedings...

\section{Camila da Silva Schmitt}

Doutoranda no Programa de Pós-Graduação em Administração (PPGA) da Universidade Federal de Santa Catarina (UFSC) (2018-2022). Mestre em Administração, linha de pesquisa em Marketing (2014) e graduada em Administração com habilitação em Comércio Exterior (2011) pela Fundação Universidade Regional de Blumenau (FURB). Professora Universitária da FURB, lecionando atualmente Introdução ao Comércio Exterior, Normas de Importação e Exportação para o curso de Tecnologia em Comércio Exterior. Docência na Universidade do Vale do Itajaí (UNIVALI), responsável no Campus Itajaí pela disciplina Marketing Digital no curso de Administração matutino e noturno e no Campus Balneário Camboriú pela temática de Fundamentos de Marketing e Marketing Digital no curso de CST em Tecnologia em Marketing. Professora também na PósGraduação de Marketing Digital, com as disciplinas: Introdução ao Marketing Digital e Consultoria em Marketing Digital e na Pós-Graduação de Comportamento Humano no Trabalho, com a disciplina: Endomarketing. E-mail: camila.s.schmitt@gmail.com.

\section{Martin de La Martinière Petroll}

Professor Adjunto C3 do Departamento de Ciências da Administração do Centro Socioeconômico da Universidade Federal de Santa Catarina (CAD/CSE/UFSC). Doutor (2013) em Administração pelo Programa de Pós-Graduação em Administração da Universidade Federal do Paraná (UFPR) com período de doutorado-sanduíche na Boston College (Boston/MA/EUA). Mestre (2007) em Administração pelo Programa de Pós-Graduação em Administração da Universidade Federal do Rio 
Grande do Sul (UFRGS). Graduado (2003) em Administração de Empresas pela Universidade do Vale do Rio dos Sinos (UNISINOS). Professor e orientador de mestrado e doutorado no Programa de Pós-Graduação em Administração (PPGAdm/UFSC) e do Curso de Graduação em Administração no Departamento de Ciências da Administração (CAD-UFSC). Linha de pesquisa: Marketing, Comportamento do Consumidor, Varejo, Comunicação. Autor de diversos artigos publicados em revistas científicas e anais de eventos nacionais e internacionais. Líder do grupo de pesquisa registrado na UFSC e no CNPq, denominado: Núcleo de Inteligência Competitiva Organizacional em Marketing e Logística (NICO). E-mail: martin.petroll@ufsc.br.

Recebido em: 30.01.2018

Aprovado em: 26.08.2020 